\title{
Safety and efficacy of multiband mucosectomy for Barrett's esophagus: a systematic review with pooled analysis
}

\author{
Marco Spadaccinia,b, Paul J. Belletruttic, Simona Attardo d, Roberta Masellia, \\ Viveksandeep Thoguluva Chandrasekare, Piera Alessia Galtieria, Alessandro Fugazza ${ }^{a}$, \\ Andrea Anderloni ${ }^{a}$, Silvia Carrara ${ }^{a}$, Gaia Pellegattaa ${ }^{a}$ Cesare Hassan ${ }^{f}$, Prateek Sharma ${ }^{e}$, Alessandro Repicia, \\ Humanitas Research Hospital, MI, Italy; Humanitas University, MI, Italy; University of Calgary, Canada; AOU \\ Ospedale Maggiore della Carità, Novara, Italy; Kansas City VA Medical Center, USA; Nuovo Regina Margherita \\ Hospital, Rome, Italy
}

\section{Abstract}

Background According to guidelines, all visible lesions in Barrett's esophagus (BE) should be endoscopically resected. Available methods of endoscopic resection include the cap-assisted technique and, more recently, multiband mucosectomy (MBM). Data on the efficacy and safety of MBM have yet to be systematically reviewed. We performed the first systematic review with pooled analysis to evaluate the outcomes of MBM in patients with BE.

Methods Electronic databases (Medline, Scopus, EMBASE) were searched up to August 2019. Studies including patients with BE who underwent MBM were eligible. The primary outcome was the adverse events rate. Secondary outcomes were the proportions of complete resections and $\mathrm{R} 0$ resections. Outcomes were assessed by pooling data using a random or fixed-effect model, according to the degree of heterogeneity, to obtain a proportion with a $95 \%$ confidence interval.

Results Fourteen studies were eligible (1334 procedures, 986 patients). The adverse event rate was $5.3 \%$. Immediate and post-procedural bleeding, perforations and strictures occurred in $0.2 \%, 0.7 \%, 0.3 \%$ and $3.9 \%$ of procedures, respectively. Focal lesions were resected at a complete rate of $97.6 \%$ with an R0 resection rate of $94.1 \%$.

Conclusion MBM is a safe and effective technique for treating visible lesions in BE.

Keywords Endoscopy, foregut, adenocarcinoma

Ann Gastroenterol 2021; 35 (3): 1-13

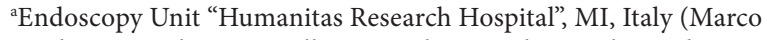
Spadaccini, Roberta Maselli, Piera Alessia Galtieri, Alessandro Fugazza, Andrea Anderloni, Silvia Carrara, Gaia Pellegatta,

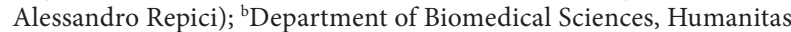
University, MI, Italy (Marco Spadaccini, Alessandro Repici); ${ }^{c}$ Medicine Department, University of Calgary, Calgary, Canada (Paul J. Belletrutti); ' $\mathrm{d}$ astroenterology Unit "AOU Ospedale Maggiore della Carità", Novara, Italy (Simona Attardo); ${ }^{\mathrm{e} G a s t r o e n t e r o l o g y ~ a n d ~}$ Hepatology "Kansas City VA Medical Center", USA (Viveksandeep Thoguluva Chandrasekar, Prateek Sharma); ${ }^{\mathrm{f}}$ Digestive Endoscopy Unit, Nuovo Regina Margherita Hospital, Rome, Italy (Cesare Hassan)

Conflict of Interest: None
\end{abstract}

Correspondence to: Marco Spadaccini, MD, Digestive Endoscopy Unit, Via Manzoni 56, 20089 Rozzano, Milan, Italy, e-mail: marcospadaccini9@gmail.com

Received 19 November 2020; accepted 7 February 2021; published online 2 April 2021

DOI: https://doi.org/10.20524/aog.2021.0620

\section{Introduction}

The incidence of esophageal adenocarcinoma (EAC) has been sharply increasing over the past several decades [1]. In the past, despite significant procedure-related morbidity and mortality, a radical surgical approach was the standard of care for Barrett's esophagus (BE) with high-grade dysplasia (HGD) or early EAC (T1). However, in recent times, endoscopic management has become a viable approach for this subgroup of patients. Moreover, these minimally invasive endoscopic techniques have been demonstrated to achieve similar oncological outcomes yet confer less risk compared to traditional surgery [2]. The current consensus states that any visible lesion in the context of $\mathrm{BE}$ should be endoscopically resected, because of its higher likelihood of containing histologically significant pathology, namely HGD or early carcinoma [2]. Conversely, extended resection aiming at complete metaplasia eradication should not be preferred over ablation strategies owing to 
the higher risk of stricturing [3-5]. Current endoscopic methods of mucosal resection (EMR) include the lift-andcut cap-assisted technique and, more recently, the multiband mucosectomy (MBM) technique. The cap-assisted procedure is more technically challenging. Furthermore, submucosal lifting is required for every resection, as well as the delicate repositioning of the snare within the cap each time. Alternatively, $\mathrm{MBM}$ is performed with a device derived from a variceal ligator: the target lesion is suctioned into the ligation cap and a rubber band is released to create a pseudopolyp; then the lesion is resected with a hot snare without the prior requirement of submucosal lifting. MBM has already been demonstrated in previous studies to be as safe and effective as the cap-assisted technique, showing shorter procedure times and lower costs. Since its first description, several prospective and retrospective series on MBM have been published, but data on the efficacy and safety of MBM have never been systematically reviewed. We performed a pooled analysis to evaluate the safety and efficacy of MBM in patients with advanced BE.

\section{Materials and methods}

This systematic review with pooled analysis was conducted in accordance with the Preferred Reporting Items for Systematic Reviews and Meta-Analysis (PRISMA) guidelines [6]. Complete information about sources and search strategy, selection process, data extraction and quality assessment are reported in Appendix A.

\section{Inclusion and exclusion criteria}

For the purposes of our systematic review, we evaluated for inclusion all clinical studies that met the following criteria: 1) studies including patients with visible lesions in $B E$ who underwent endoscopic resection by the MBM technique; and 2) studies including patients who underwent endoscopic resection by the MBM technique as part of a metaplasia/ dysplasia eradication protocol. The inclusion process was not limited by the study design, the exact device used or adjunctive ablative treatment.

The following exclusion criteria were applied: 1) studies not published as full text articles; 2) studies not published in the English language; 3 ) studies including less than 10 patients; and 4) studies including patients with BE who underwent EMR if outcomes reports were not sub-categorized according to EMR technique (cap-assisted EMR, lift and cut, MBM).

\section{Outcomes and definitions}

The primary outcome was the rate of adverse events, namely intra- and postprocedural bleeding, perforation and stricture. Mild intraprocedural bleedings, endoscopically managed during the procedure and not affecting its completeness, were tabulated, but not considered as adverse events. Strictures were considered relevant only if the narrowing of the lumen became symptomatic and required endoscopic or surgical treatment.

Secondary outcomes were focused on MBM performed for resecting visible lesions only. The ratios of complete resection and $\mathrm{R} 0$ resection were assessed when such date were available for extraction. The endoscopic resection was considered macroscopically complete if the visible lesion was reported by the endoscopist to have been entirely removed. The absence of neoplastic invasion at the deep and lateral margins of the resected specimens was considered as an R0 resection for lesions resected in an en bloc fashion. In cases of piecemeal resections, R0 at the deep margin of all resected specimens derived from a single lesion was required.

\section{Statistical analysis}

Measures of the effect of interest were pooled ratios in the form of percentages or frequencies of events over the total number of patients for each outcome. Pooled ratios were expressed as proportions of patients (\%) with 95\% confidence limits and heterogeneity $\left(I^{2}\right)$. A P-value of $<0.05$ was considered statistically significant. The random effects model described by DerSimonian and Laird was also used for the analysis. Corresponding forest plots were constructed for both the primary and secondary outcomes. The weights of individual studies were represented by the individual squares. Heterogeneity between studies was assessed using the inconsistency index $\left(I^{2}\right)$ with $I^{2}$-values of $0-30 \%, 30-60 \%$, $60-75 \%$ and $75-100 \%$ suggestive of low, moderate, substantial and considerable heterogeneity, respectively. All meta-analytic computations, including the estimates and 95\% confidence intervals for pooled ratios as well as the heterogeneity ( $I^{2}$ statistics), were performed using statistical software Open Meta analyst (CEBM, Brown University, Rhode Island, USA).

\section{Results}

\section{Study characteristics and quality}

The literature search results in 964 articles (Fig. 1). After a review of titles and abstracts, 36 studies were considered as full text. Fourteen studies finally matched the selection criteria and were deemed eligible for the analysis [7-20]. The included studies were published between 2006 and 2019. All studies but 3 were performed in Europe; the remainder were from North America. Four studies $[12,13,15,17]$ had a prospective design and the other 10 were retrospective [7-11,14,16,18-20]. Eight studies were single-center experiences $[11,13,15,17]$. The Newcastle-Ottawa Scale [21] for the methodology quality assessment of non-randomized studies resulted in a mean score of 5.4 (range 5-6) (Appendix B, Supplementary Table 1). Characteristics of the included studies are reported in Table 1.

We provide data on $1334 \mathrm{MBM}$ procedures performed on 986 patients (mean age: $68.4 \pm 2.8$ years), of whom $84.6 \%$ 
were male (9 studies [7,9-12,14-16,20]). The indications for endoscopic resection varied across the studies, with $62.9 \%$ of procedures being performed in order to resect focal lesions and the remaining $37.1 \%$ in the context of metaplasia/ dysplasia eradication protocols. In 9.4\%, patients had undergone previous endoscopic resections (data provided by 5 studies $[8,14,16,19], 557$ procedures). Visible lesion characteristics are provided in Appendix B, Supplementary Table 2. Considering the histological findings, $31.3 \%$ of the lesions were reported to be HGD and 52.2\% were adenocarcinomas (7 studies [7,9,11,12,16,18,20]).

\section{Primary outcomes: adverse events}

The overall adverse event rate, pooled from the reports of all 14 studies, was $5.3 \%$ ( $I^{2}=83.4 \%$; see Fig. 2$)$. Immediate and postprocedural bleeding occurred in $0.2 \%\left(I^{2}=0 \%\right)$ and $0.7 \%$ $\left(I^{2}=0 \%\right)$ of procedures, respectively. During 99 out of 1334

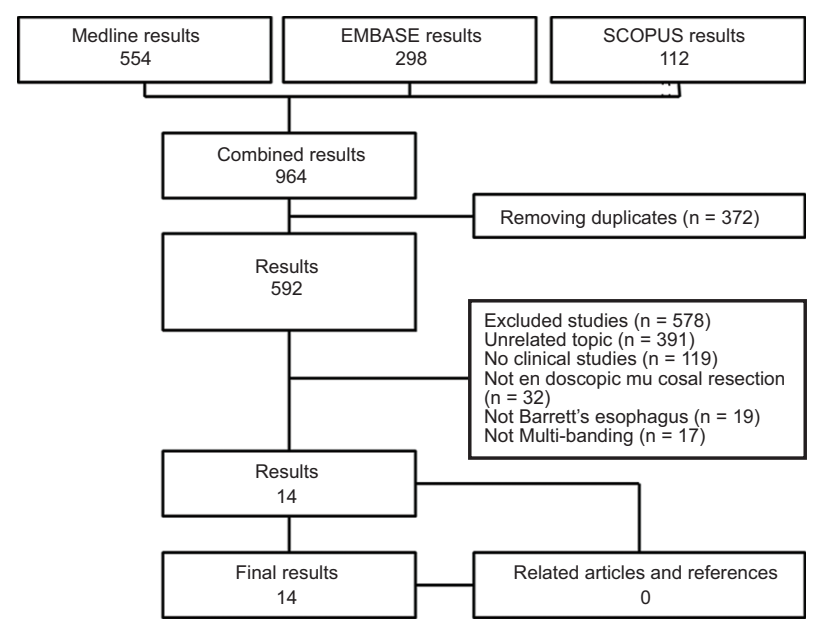

Figure 1 PRISMA flow chart
(7.4\%) procedures, mild bleeding not affecting the procedural completeness was reported. Perforations and strictures were reported at a rate of $0.3 \%\left(I^{2}=0 \%\right.$, see Fig. 3$)$ and $3.9 \%$ $\left(I^{2}=80.4 \%\right)$, respectively. Safety outcomes of each study are provided in Table 2.

\section{Sensitivity analysis}

A sensitivity analysis focused on focal lesion resection (584 procedures) reported an overall adverse event rate of $5.5 \%$ with a low level of heterogeneity $\left(I^{2}=0 \%\right.$; Supplementary Fig. 1., Appendix C).

Ratios of bleeding, perforation and stricture were 1.6\%, $1 \%$ and 3.3\%, respectively (Supplementary Figs. 2-4, Appendix C). Likewise, a low level of heterogeneity was reported $\left(I^{2}=0 \%\right)$. Results of the sensitivity analysis focused on prospective studies (453 procedures) are provided in Appendix C (Supplementary Figs. 5-10).

\section{Secondary outcomes: efficacy}

A pooled rate of $97.6 \%\left(I^{2}=49.9 \%\right)$ for complete resection was calculated from data across 7 studies $[8,9,11,12,14-16]$ (Supplementary Fig. 11, Appendix C). Only 32.2\% of the treated lesions were resected in an en bloc fashion $\left(I^{2}=0 \%\right.$; Supplementary Fig. 12, Appendix C), with a mean number of specimens per procedure of $4.2 \pm 1.6$. The mean diameter of resected specimens was $15.4 \pm 2.1 \mathrm{~mm}$ according to data reported by 5 studies $[7,8,12,14,15]$. The $\mathrm{R} 0$ resection rate pooled from 7 studies was $94.1 \%$ ( $95 \%$ confidence interval 89.9-98.4\%; $I^{2}=81.7 \%$; Supplementary Fig. 13, Appendix C) $[8,9,12,14-16,20]$. Data on procedural time were reported by only 3 studies $[12,15,16]$, with a pooled time of $22.9 \pm 9.7 \mathrm{~min}$ per visible lesion resected. Efficacy outcomes of each study are provided in Appendix B, Supplementary Table 3.

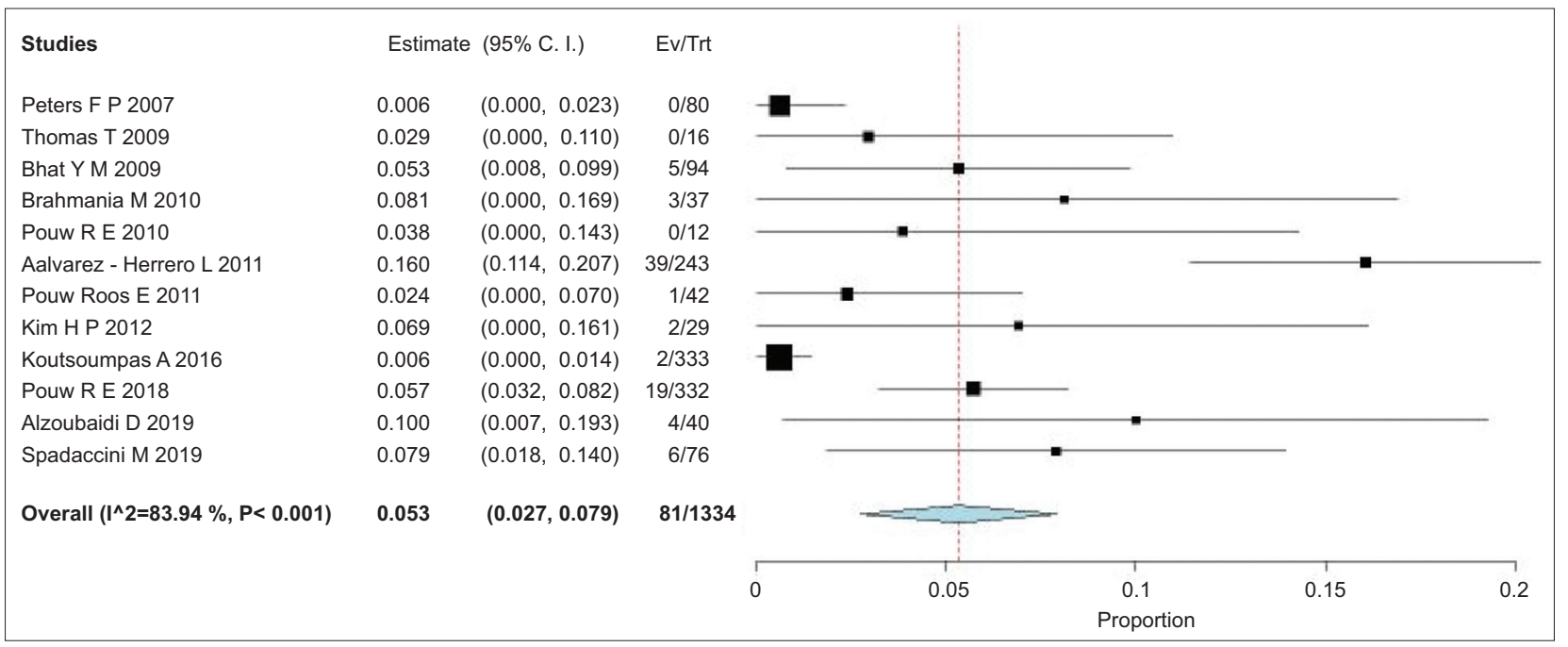

Figure 2 Forest plot: overall adverse events rate CI, confidence interval 


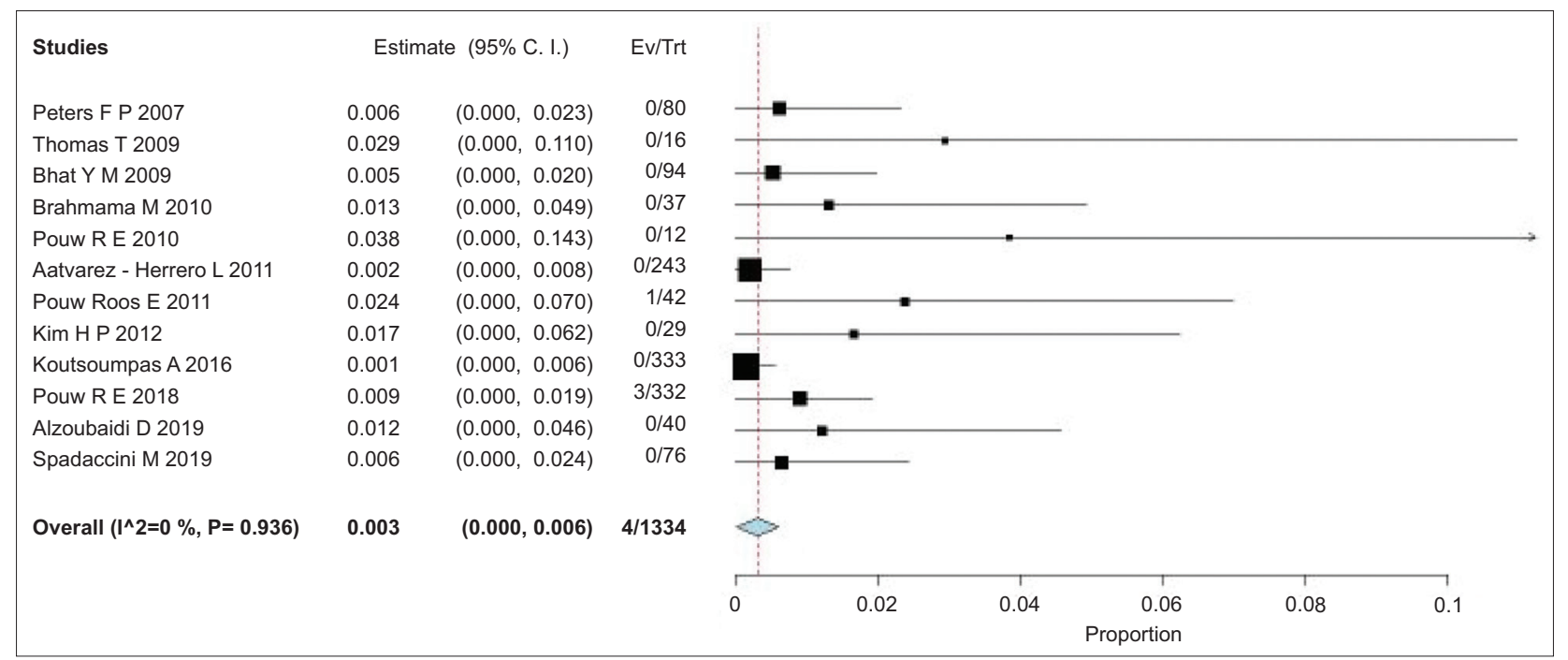

Figure 3 Forest plot: Perforation rate CI, confidence interval

\section{Discussion}

MBM is the most commonly used technique for endoscopic resection in $\mathrm{BE}$. This is the first pooled analysis confirming MBM to be safe for endoscopic resection of superficial Barrett's neoplasia. Our results are relevant for the following reasons. First of all, serious procedure-related adverse events, such as perforations or severe bleedings, are quite uncommon and large studies assessing an accurate estimation of such complications are still lacking. Pooling data from more than 1300 procedures provides the most feasible and reliable estimation based on the current literature. Secondly, when pooling data in terms of safety, the low level of heterogeneity across the studies is even more relevant when one considers that American and European settings were involved in all the studies included in our analysis. This made our results generalizable where BE is most diffuse and our conclusions stronger.

According to our analysis, the most common event is intraprocedural bleeding, with a pooled rate of $7.4 \%$. In all cases, however, it appears that such bleeding did not affect the completeness of the procedure. Some may consider this type of bleeding to be part and parcel of performing mucosal resection. As such, this degree of bleeding is probably underreported in the literature, particularly from retrospective series. As a matter of fact, the highest rate of such bleeding was reported in the recent 2018 international multicenter by Pouw et al. Intraprocedural bleeding occurred in 48 of 332 procedures (14\%), but hemostasis was achieved in all cases using a variety of modalities including snare tip, grasper coagulation, argon plasma coagulation, and/or hemoclip placement.

More serious intraprocedural bleeding preventing completion of the resection occurred at a pooled rate of $0.2 \%$. Clinically significant postprocedural bleeding was also a rare occurrence, at $0.7 \%$ ( 15 total events) across the 13 studies. In these instances, either supportive care or additional endoscopy was required to control hemorrhage from the resection site. Surgical or radiological intervention was not required in any cases.
As for the more feared complication of perforation, most series recorded no perforations as a result of MBM (11 studies) and the perforations reported in the remaining 2 studies yielded a pooled rate of $0.3 \%$ ( 4 total events). In the Pouw et al 2011 randomized study comparing cap-assisted resection and MBM using the Duette system (Cook Endoscopy, Limerick, Ireland), 1 perforation occurred in the MBM group (compared to 3 perforations in the cap-assisted group, P-value not significant). However, the defect in this case could not be closed endoscopically and the patient was referred for surgery. The other 3 perforations were reported in the recent 2018 multicenter MBM study in which Pouw et al evaluated the new Captivator device (Boston Scientific Corp, Marlborough, MA, USA). In 2 of them, perforation was recognized intraprocedurally and the defect was closed with hemoclips (1 with the addition of an endoloop around the clips). Both patients were observed in hospital without any further sequelae. In the third instance, the resection defect had been closed prophylactically with clips but subcutaneous emphysema was recognized postprocedurally and a contrast esophagram showed a small leak at the site of resection. This patient was also observed in hospital and recovered uneventfully without the need for any further intervention. It is possible that unfamiliarity with the new device was related to the higher number of perforations in this trial, but the numbers are too small to draw any firm conclusions. Additionally, 2 recent retrospective, single-center series, involving 40 and 76 patients, reported no differences between the Duette and Captivator systems after comparing endoscopic outcomes and histology features $[14,16]$. Finally, we consider it remarkable that no delayed perforations have been reported in the MBM literature. This has implications for the modality of patients' management and follow up, probably meaning that, once the procedure has been successfully completed, there is no need for hospitalization as well as early postprocedural strict follow up. Likewise, no deaths have occurred.

Although these data are reassuring as regards the safety of MBM, they all came from tertiary referral centers. Hence, 
in order to achieve such safety outcomes, it is clear that any endoscopist who performs band mucosectomy must be very adept at managing adverse events such as intra/postprocedural bleeding, and must also be able to recognize and reliably close small esophageal perforations. This strengthens the argument that mucosal resection of Barrett's neoplasia is best performed at high volume referral centers by experienced endoscopists.

Esophageal strictures can occur as a result of band mucosectomy. The frequency varies widely in the reported literature, but can approach $40-50 \%$ if extensive EMR is performed as a Barrett's eradication protocol [4,5]. In this meta-analysis, the pooled rate for the development of a symptomatic stricture post-MBM performed for resecting visible lesions was $3.3 \%$, with no heterogeneity across the studies. In all patients, endoscopic dilation allowed a successful management of strictures, with all patients being able to resume a regular diet. For comparison, a recent metaanalysis of the outcomes of endoscopic submucosal dissection for Barrett's neoplasia reported a pooled esophageal stricture rate of $11.3 \%$ [5]. Nonetheless, the low pooled rate of strictures in this meta-analysis, compared to the historical literature on Barrett's EMR, suggests that band mucosectomy may be less prone to subsequent esophageal stenosis and, in any event, no strictures refractory to endoscopic intervention were reported. This favorable outcome in terms of stricture developments may be related to the MBM technique, which normally does not achieve very deep tissue resection, preserving part of the submucosa.

This meta-analysis also confirmed the high clinical efficacy of MBM, as the pooled complete resection rate was $97.6 \%$ with a pooled $\mathrm{R} 0$ resection rate of $94.1 \%$. However, these data should be interpreted with caution, since significant heterogeneity was reported.

This meta-analysis has several limitations. First, it did not address long-term follow-up outcomes or the rate of neoplasia recurrence; however, the role of EMR techniques in managing neoplastic BE has already been assessed by several highquality studies [2]. Secondly, the patient populations varied across studies, and factors such as comorbidity burden and anticoagulant/antiplatelet use would be expected to affect safety outcomes. Moreover, the MBM technique is not standardized worldwide, and factors such as the length and surface area resected, as well as the number of pieces resected per session, could also impact safety and efficacy outcomes. Of course, the burden of such clinical and technical variables needs to be evaluated in future well-powered studies. However, being conclusively reassuring about the safety profile of the MBM approach, our study may be informative for designing such studies. Finally, the overall quality of the studies is limited, given that most were non-randomized and retrospective in nature. However, the low-to-moderate inter-study heterogeneity across the studies reported for the main outcomes offers reassurance as to the reliability of our estimates. This provides endoscopists with a hitherto missing tool, so they may better counsel patients about the safety of the most diffuse approach for treating neoplastic BE.

In conclusion, based on the available data, this metaanalysis confirms the safety and efficacy of MBM for the treatment of advanced Barrett's neoplasia. Although infrequent, serious adverse events can occur; thus, any endoscopy center performing MBM must have the experience and expertise to manage complications.

\section{Summary Box}

\section{What is already known:}

- The incidence of esophageal adenocarcinoma has been sharply increasing over the past several decades

- Endoscopic management have been demonstrated to achieve similar oncological outcomes in early esophageal adenocarcinoma, yet confer less risk compared to traditional surgery

- The multiband mucosectomy (MBM) technique has been proposed as a viable modality for performing endoscopic mucosal resection in Barrett's esophagus

\section{What the new findings are:}

- MBM proved its safety over more than 1300 procedures, with around a $1 \%$ risk of perforation

- Target lesions were resected in almost $100 \%$ of cases, underlying the feasibility of such an approach

- Further studies are needed to assess long-term outcomes

\section{References}

1. Hur C, Miller M, Kong CY, et al. Trends in esophageal adenocarcinoma incidence and mortality. Cancer 2013;119:11491158.

2. Weusten B, Bisschops R, Coron E, et al. Endoscopic management of Barrett's esophagus: European Society of Gastrointestinal Endoscopy (ESGE) Position Statement. Endoscopy 2017;49:191198.

3. Lewis JJ, Rubenstein JH, Singal AG, Elmunzer BJ, Kwon RS, Piraka CR. Factors associated with esophageal stricture formation after endoscopic mucosal resection for neoplastic Barrett's esophagus. Gastrointest Endosc 2011;74:753-760.

4. Chennat J, Konda VJ, Ross AS, et al. Complete Barrett's eradication endoscopic mucosal resection: an effective treatment modality for high-grade dysplasia and intramucosal carcinoma-an American single-center experience. Am J Gastroenterol 2009;104:2684-2692.

5. Yang D, Zou F, Xiong S, Forde JJ, Wang Y, Draganov PV. Endoscopic submucosal dissection for early Barrett's neoplasia: a meta-analysis. Gastrointest Endosc 2018;87:1383-1393.

6. Shamseer L, Moher D, Clarke M, et al. Preferred reporting items for systematic review and metaanalysis protocols (PRISMA-P) 2015: elaboration and explanation. BMJ 2015;350:g7647.

7. Soehendra N, Seewald S, Groth S, et al. Use of modified multiband ligator facilitates circumferential EMR in Barrett's esophagus (with 
video). Gastrointest Endosc 2006;63:847-852.

8. Peters FP, Kara MA, Curvers WL, et al. Multiband mucosectomy for endoscopic resection of Barrett's esophagus: feasibility study with matched historical controls. Eur J Gastroenterol Hepatol 2007;19:311-315.

9. Thomas T, Singh R, Ragunath K. Trimodal imaging-assisted endoscopic mucosal resection of early Barrett's neoplasia. Surg Endosc 2009;23:1609-1613.

10. Bhat YM, Furth EE, Brensinger CM, Ginsberg GG. Endoscopic resection with ligation using a multi-band mucosectomy system in Barrett's esophagus with high-grade dysplasia and intramucosal carcinoma. Therap Adv Gastroenterol 2009;2:323-330.

11. Alvarez Herrero L, Pouw RE, van Vilsteren FG, et al. Safety and efficacy of multiband mucosectomy in 1060 resections in Barrett's esophagus. Endoscopy 2011;43:177-183.

12. Pouw RE, van Vilsteren FG, Peters FP, et al. Randomized trial on endoscopic resection-cap versus multiband mucosectomy for piecemeal endoscopic resection of early Barrett's neoplasia. Gastrointest Endosc 2011;74:35-43.

13. Pouw RE, Seewald S, Gondrie JJ, et al. Stepwise radical endoscopic resection for eradication of Barrett's oesophagus with early neoplasia in a cohort of 169 patients. Gut 2010;59:1169-1177.

14. Alzoubaidi D, Graham D, Bassett P, et al. Comparison of two multiband mucosectomy devices for endoscopic resection of Barrett's esophagus-related neoplasia. Surg Endosc 2019;33:36653672.

15. Pouw RE, Beyna T, Belghazi K, et al. A prospective multicenter study using a new multiband mucosectomy device for endoscopic resection of early neoplasia in Barrett's esophagus. Gastrointest Endosc 2018;88:647-654.

16. Spadaccini M, Bhandari P, Maselli R, et al. Multi-band mucosectomy for neoplasia in patients with Barrett's esophagus: in vivo comparison between two different devices. Surg Endosc 2020;34:3845-3852.

17. Phoa KN, Pouw RE, Bisschops R, et al. Multimodality endoscopic eradication for neoplastic Barrett oesophagus: results of an European multicentre study (EURO-II). Gut 2016;65:555-562.

18. Koutsoumpas A, Wang LM, Bailey AA, et al. Non-radical, stepwise complete endoscopic resection of Barrett's epithelium in short segment Barrett's esophagus has a low stricture rate. Endosc Int Open 2016;4:E1292-E1297.

19. Kim HP, Bulsiewicz WJ, Cotton CC, et al. Focal endoscopic mucosal resection before radiofrequency ablation is equally effective and safe compared with radiofrequency ablation alone for the eradication of Barrett's esophagus with advanced neoplasia. Gastrointest Endosc 2012;76:733-739.

20. Brahmania M, Lam E, Telford J, Enns R. Endoscopic mucosal resection: early experience in British Columbia. Can J Gastroenterol 2010;24:239-244.

21. Wells G, Shea B, O'Connell D, et al. The Newcastle-Ottawa Scale (NOS) for assessing the quality of nonrandomized studies in meta-analyses. 2013. Available from: http://www.ohri.ca/ programs/clinical_epidemiology/oxford.asp [Accessed 12 March 2021]. 


\section{Supplementary material}

\section{Appendix A}

\section{Data sources and search strategy}

We performed a comprehensive literature search in PubMed, EMBASE and SCOPUS (up to September 2019) electronic databases to identify studies evaluating the efficacy and safety of the multi-band mucosectomy technique for endoscopic mucosal resection in Barrett's esophagus. PROSPERO was searched for ongoing or recently completed systematic reviews. Electronic searches were supplemented by manual searches of references of included studies and review articles.

We identified studies using the following medical subject headings (MeSH) and the keywords "Multi-band Mucosectomy", "Multi-Banding", "Multi-band", "Banding", "Band", "Endoscopic Mucosal Resection", "EMR", "Barrett's", and "Barrett's esophagus". The search was restricted to the English language.

The Medline search string was: "((()((“multiple chronic conditions"[MeSH Terms] OR ("multiple"[All Fields] AND "chronic"[All Fields] AND "conditions"[All Fields]) OR "multiple chronic conditions"[All Fields] OR "multi"[All Fields]) AND ("Band"[Journal] OR "band"[All Fields]) AND mucosectomy[All Fields] AND barrett's[All Fields]) OR (("multiple chronic conditions" [MeSH Terms] OR ("multiple"[All Fields] AND "chronic"[All Fields] AND "conditions"[All Fields]) OR "multiple chronic conditions"[All Fields] OR "multi"[All Fields]) AND ("Band"[Journal] OR "band"[All Fields]) AND ("Empir Musicol Rev"[Journal] OR "emr"[All Fields]) AND barrett’s[All Fields])) OR (("multiple chronic conditions"[MeSH Terms] OR ("multiple"[All Fields] AND "chronic"[All Fields] AND "conditions"[All Fields]) OR "multiple chronic conditions"[All Fields] OR "multi"[All Fields]) AND ("Band"[Journal] OR "band"[All Fields]) AND barrett's[All Fields])) OR (multi-band[All Fields] AND mucosectomy[All Fields] AND barrett's[All Fields])) AND (multi-band[All Fields] AND barrett's[All Fields])) OR (multi-band[All Fields] AND ("Empir Musicol Rev"[Journal] OR “emr”[All Fields]) AND barrett's[All Fields])) OR ((“"endoscopic mucosal resection”[MeSH Terms] OR ("endoscopic"[All Fields] AND "mucosal”[All Fields] AND "resection"[All Fields]) OR "endoscopic mucosal resection”[All Fields]) AND Barrett's[All Fields]) OR ((“Empir Musicol Rev”[Journal] OR “emr”[All Fields]) AND Barrett's[All Fields])) OR ((“Empir Musicol Rev"[Journal] OR “emr"[All Fields]) AND Barrett's[All Fields] AND ("Band"[Journal] OR "band"[All Fields])) OR ((“endoscopic mucosal resection"[MeSH Terms] OR ("endoscopic"[All Fields] AND "mucosal"[All Fields] AND "resection"[All Fields]) OR “endoscopic mucosal resection"[All Fields]) AND
Barrett's[All Fields] AND ("Band"[Journal] OR "band"[All Fields])) OR ((“endoscopic mucosal resection”[MeSH Terms] OR ("endoscopic"[All Fields] AND "mucosal"[All Fields] AND "resection"[All Fields]) OR “endoscopic mucosal resection"[All Fields]) AND Barrett's[All Fields] AND banding[All Fields]) OR (("Empir Musicol Rev"[Journal] OR "emr" [All Fields]) AND Barrett's[All Fields] AND banding[All Fields])".

\section{Selection process}

Two review authors (MS \& SA) independently screened the titles and abstracts yielded by the search against the inclusion criteria. Full reports were obtained for all titles that appeared to meet the inclusion criteria or where there was any uncertainty. Review author pairs then screened the full text and abstract reports and decided whether these met the inclusion criteria. Disagreements were resolved through discussion among all the authors. The reasons for excluding trials were recorded. Neither of the review authors was blinded to the journal titles, or to the study authors or institutions. When there were multiple articles for a single study, we used the latest publication and supplemented it, if necessary, with data from the most complete version.

\section{Data extraction}

Using standardized forms, 2 reviewers (MS \& SA) extracted data independently and in duplicate from each eligible study. Reviewers resolved disagreements by discussion. Unresolved disagreements were resolved by 2 arbitrators (PS \& AR). The following data were extracted for each study: the publication status, the study design and location, the number of centers involved, the number of patients, patient characteristics (mean/ median age, sex), the number of procedures, indications, mean/median lesion size, lesion aspect and histology, complete resections, en bloc resections, R0 resections, and adverse events (bleedings, perforation, stricture).

\section{Quality assessment}

Quality was assessed by the modified Newcastle-Ottawa Scale for non-randomized studies, ranging from 0 (lowquality) to 5 (high-quality). Two reviewers (MS \& SA) assessed quality measures for included studies and discrepancies were adjudicated by collegial discussion. 
Supplementary Tables

\section{Appendix B}

Supplementary Table 1 Newcastle-Ottawa scale

\begin{tabular}{|c|c|c|c|c|c|c|c|c|c|}
\hline \multirow[t]{2}{*}{ Author, year [Reference] } & \multicolumn{4}{|c|}{ Selection } & \multirow{2}{*}{$\begin{array}{c}\text { Comparability } \\
1\end{array}$} & \multicolumn{3}{|c|}{ Outcome } & \multirow[t]{2}{*}{ Total } \\
\hline & 1 & 2 & 3 & 4 & & 1 & 2 & 3 & \\
\hline Soehendra 2006 [7] & 1 & 0 & 1 & 1 & 0 & 0 & 1 & 1 & 5 \\
\hline Peters 2007 [8] & 1 & 0 & 1 & 1 & 0 & 0 & 1 & 1 & 5 \\
\hline Thomas 2009 [9] & 1 & 0 & 1 & 1 & 0 & 0 & 1 & 1 & 5 \\
\hline Bhat 2009 [10] & 1 & 0 & 1 & 1 & 0 & 0 & 1 & 1 & 5 \\
\hline Brahmania 2010 [20] & 1 & 0 & 1 & 1 & 0 & 0 & 1 & 1 & 5 \\
\hline Pouw 2010 [13] & 1 & 0 & 1 & 1 & 0 & 1 & 1 & 1 & 6 \\
\hline Aalvarez-Herrero 2011 [11] & 1 & 0 & 1 & 1 & 0 & 0 & 1 & 1 & 6 \\
\hline Pouw Roos 2011 [12] & 1 & 0 & 1 & 1 & 0 & 1 & 1 & 1 & 6 \\
\hline Kim 2012 [19] & 1 & 0 & 1 & 1 & 0 & 0 & 1 & 1 & 5 \\
\hline Koutsoumpas 2016 [18] & 1 & 0 & 1 & 1 & 0 & 1 & 1 & 1 & 6 \\
\hline Phoa 2016 [17] & 1 & 0 & 1 & 1 & 0 & 1 & 1 & 1 & 6 \\
\hline Pouw 2018 [15] & 1 & 0 & 1 & 1 & 0 & 1 & 1 & 1 & 6 \\
\hline Alzoubaidi 2019 [14] & 1 & 0 & 1 & 1 & 0 & 0 & 1 & 1 & 5 \\
\hline Spadaccini 2019 [16] & 1 & 0 & 1 & 1 & 0 & 0 & 1 & 1 & 5 \\
\hline
\end{tabular}

Supplementary Table 2 Visible lesion characteristics

\begin{tabular}{|c|c|c|c|c|c|c|c|c|c|c|c|c|}
\hline \multirow{2}{*}{$\begin{array}{l}\text { Author, } \\
\text { year [Reference] }\end{array}$} & \multirow{2}{*}{$\begin{array}{c}\text { Focal } \\
\text { lesion }(\mathrm{n})\end{array}$} & \multirow{2}{*}{$\begin{array}{l}\text { Mean lesion } \\
\text { size }(\mathrm{mm})\end{array}$} & \multirow{2}{*}{$\begin{array}{l}\text { Previous } \\
\text { resections (n) }\end{array}$} & \multicolumn{9}{|c|}{ Paris classification } \\
\hline & & & & Is & Ip & Ips & IIa & $\mathrm{IIb}$ & IIc & Is+IIa & IIa+IIc & $\mathrm{IIa}+\mathrm{IIb}$ \\
\hline Soehendra 2006 [7] & 0 & 1 & 1 & 0 & 0 & 0 & 0 & 0 & 0 & 0 & 0 & 0 \\
\hline Peters 2007 [8] & 24 & 1 & 11 & 0 & 0 & 0 & 4 & 11 & 0 & 0 & 9 & 0 \\
\hline Thomas 2009 [9] & 16 & 13 & 1 & 0 & 0 & 0 & 4 & 9 & 0 & 0 & 3 & 0 \\
\hline Bhat 2009 [10] & 0 & 1 & 1 & 1 & 1 & 1 & 1 & 1 & 1 & 1 & 1 & 1 \\
\hline Brahmania 2010 [20] & 37 & 1 & 1 & 1 & 1 & 1 & 1 & 1 & 1 & 1 & 1 & 1 \\
\hline Pouw 2010 [13] & 12 & 1 & 1 & 1 & 1 & 1 & 1 & 1 & 1 & 1 & 1 & 1 \\
\hline $\begin{array}{l}\text { Aalvarez-Herrero } \\
2011 \text { [11] }\end{array}$ & 113 & 1 & 1 & 1 & 1 & 1 & 1 & 1 & 1 & 1 & 1 & 1 \\
\hline Pouw Roos 2011 [12] & 42 & 1 & 1 & 1 & 1 & 1 & 1 & 1 & 1 & 1 & 1 & 1 \\
\hline Kim 2012 [19] & 29 & 1 & 0 & 1 & 1 & 1 & 1 & 1 & 1 & 1 & 0 & 0 \\
\hline $\begin{array}{l}\text { Koutsoumpas } \\
2016 \text { [18] }\end{array}$ & 118 & 1 & 1 & 1 & 1 & 1 & 1 & 1 & 1 & 1 & 1 & 1 \\
\hline Phoa 2016 [17] & 1 & 1 & 1 & 1 & 1 & 1 & 1 & 1 & 1 & 1 & 1 & 1 \\
\hline Pouw 2018 [15] & 332 & 10 & 0 & 6 & 6 & 0 & 217 & 68 & 12 & 0 & 0 & 0 \\
\hline Alzoubaidi 2019 [14] & 40 & 13,5 & 0 & 1 & 1 & 0 & 31 & 3 & 0 & 0 & 3 & 0 \\
\hline Spadaccini 2019 [16] & 76 & 13,5 & 23 & 19 & 0 & 0 & 29 & 13 & 6 & 1 & 1 & \\
\hline
\end{tabular}


Supplementary Table 3 Efficacy outcomes

\begin{tabular}{|c|c|c|c|c|c|c|c|}
\hline $\begin{array}{l}\text { Author, } \\
\text { year [Reference] }\end{array}$ & $\begin{array}{l}\text { Focal lesions } \\
\text { (n) }\end{array}$ & $\begin{array}{l}\text { Specimens } \\
\text { per procedure } \\
(\text { mean } \mathrm{n})\end{array}$ & $\begin{array}{c}\text { Specimen } \\
\text { diameter } \\
(\text { mean } \mathrm{mm})\end{array}$ & $\begin{array}{l}\text { Procedural time } \\
\quad(\text { mean min) }\end{array}$ & $\begin{array}{l}\text { Complete } \\
\text { resections } \\
\text { (n) }\end{array}$ & $\begin{array}{l}\text { En bloc } \\
\text { resections } \\
\text { (n) }\end{array}$ & $\begin{array}{l}\text { R0 resections } \\
\text { (n) }\end{array}$ \\
\hline $\begin{array}{l}\text { Soehendra } \\
2006[7]\end{array}$ & 0 & 1 & 14.3 & 1 & 1 & 0 & 1 \\
\hline Peters 2007 [8] & 24 & 6 & 17 & 1 & 24 & 1 & 24 \\
\hline Thomas 2009 [9] & 16 & 1 & 1 & 1 & 14 & 1 & 12 \\
\hline Bhat 2009 [10] & 0 & 1 & 1 & 1 & 1 & 1 & 1 \\
\hline $\begin{array}{l}\text { Brahmania } \\
2010[20]\end{array}$ & 37 & 6 & 1 & 1 & 1 & 1 & 21 \\
\hline Pouw 2010 [13] & 12 & 1 & 1 & 1 & 1 & 1 & 1 \\
\hline $\begin{array}{l}\text { Aalvarez-Herrero } \\
2011 \text { [11] }\end{array}$ & 113 & 3 & 1 & 1 & 103 & 32 & 1 \\
\hline $\begin{array}{l}\text { Pouw Roos } \\
2011 \text { [12] }\end{array}$ & 42 & 5 & 18 & 34 & 42 & 1 & 42 \\
\hline Kim 2012 [19] & 29 & 1 & 1 & 1 & 1 & 1 & 1 \\
\hline $\begin{array}{l}\text { Koutsoumpas } \\
2016 \text { [18] }\end{array}$ & 118 & 1 & 1 & 1 & 1 & 1 & 1 \\
\hline Phoa 2016 [17] & 1 & 1 & 1 & 1 & 1 & 1 & 1 \\
\hline Pouw 2018 [15] & 332 & 1 & 15 & 16 & 322 & 111 & 325 \\
\hline $\begin{array}{l}\text { Alzoubaidi } \\
2019 \text { [14] }\end{array}$ & 40 & 2.7 & 12.9 & 1 & 40 & 1 & 36 \\
\hline $\begin{array}{l}\text { Spadaccini } \\
2019 \text { [16] }\end{array}$ & 76 & 2.6 & 1 & 18.7 & 76 & 25 & 74 \\
\hline
\end{tabular}




\section{Supplementary Figures}

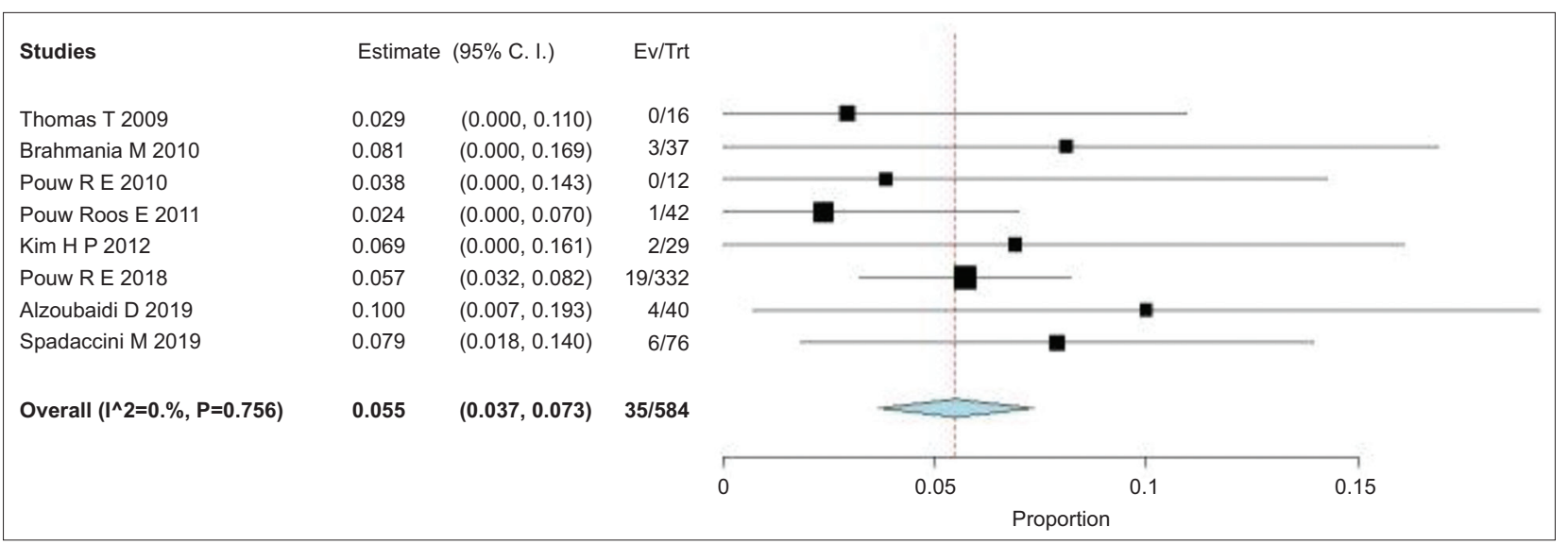

Supplementary Figure 1 Sensitivity analysis focused on focal lesion resection: overall adverse event rate (Forest plot) CI, Confidence Interval; Ev, events; Trt, treatments

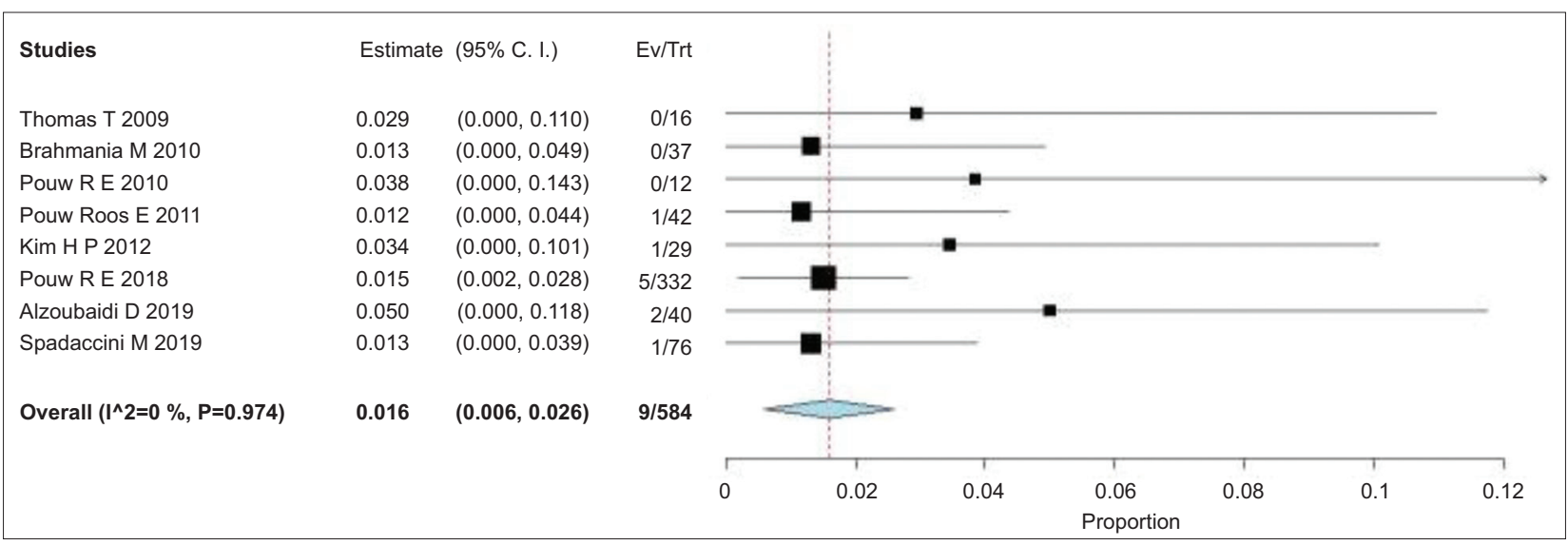

Supplementary Figure 2 Sensitivity analysis focused on focal lesion resection: bleeding rate (Forest plot) CI, Confidence Interval; Ev, events; Trt, treatments

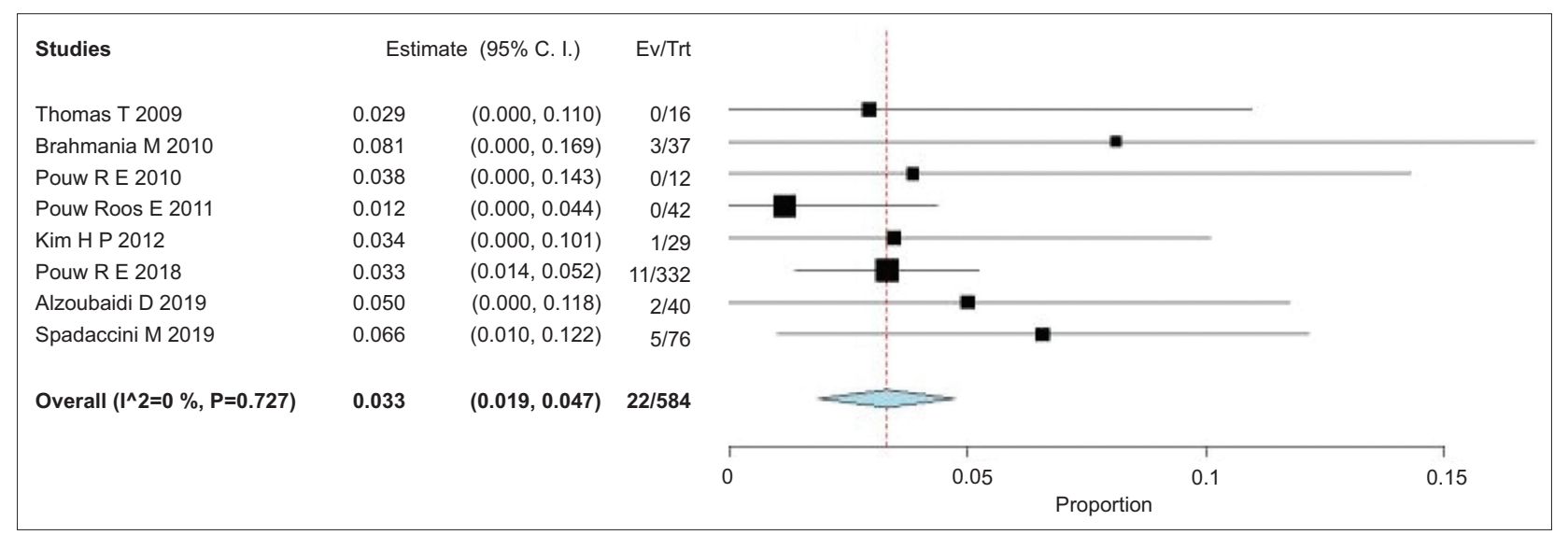

Supplementary Figure 3 Sensitivity analysis focused on focal lesion resection: perforation rate (Forest plot) CI, Confidence Interval; Ev, events; Trt, treatments 


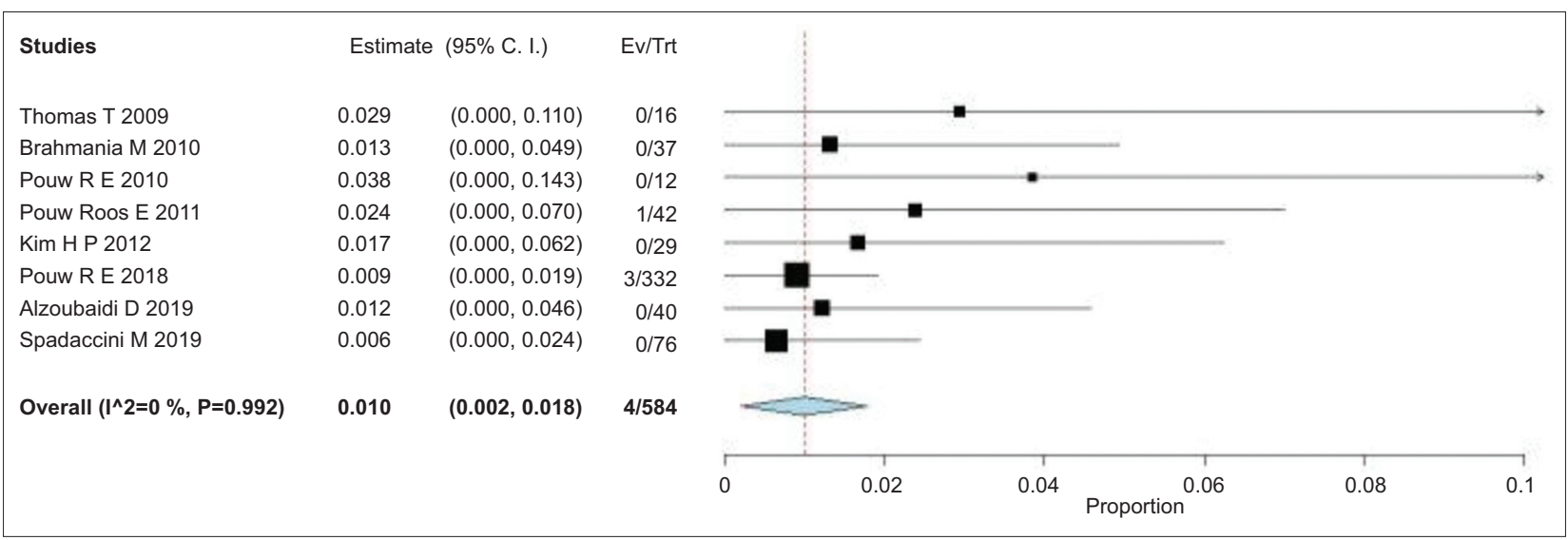

Supplementary Figure 4 Sensitivity analysis focused on focal lesion resection: stricture rate (Forest plot) CI, Confidence Interval; Ev, events; Trt, treatments

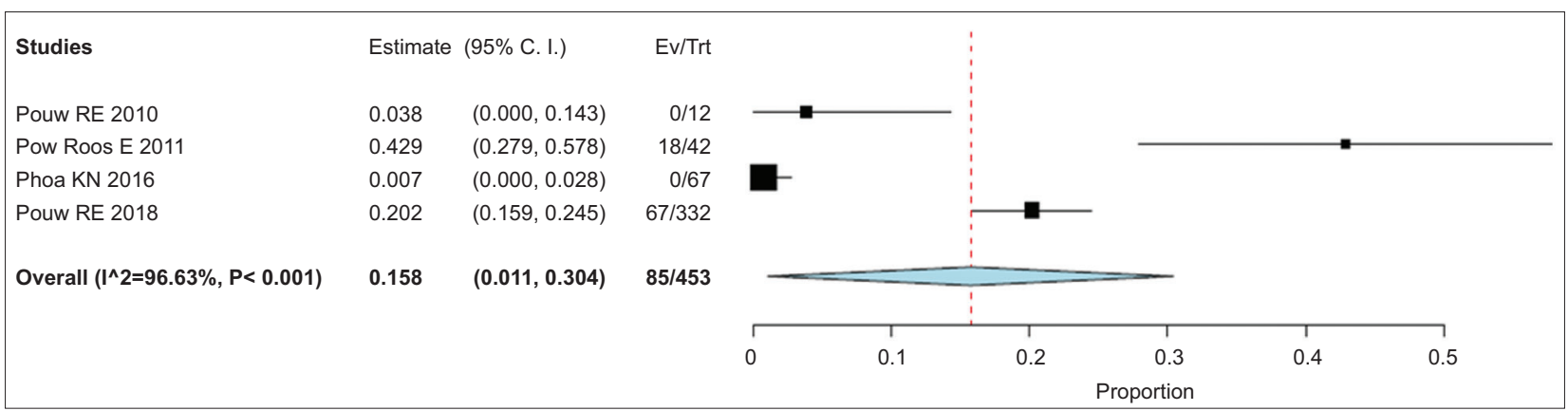

Supplementary Figure 5 Sensitivity analysis focused on prospective studies: overall adverse event rate (Forest plot)

CI, Confidence Interval; Ev, events; Trt, treatments

\begin{tabular}{|c|c|c|c|c|c|c|c|c|c|}
\hline Studies & Estimate & (95\% C. I.) & Ev/Trt & & & & & & \\
\hline Pouw RE 2010 & 0.038 & $(0.000,0.143)$ & $0 / 12$ & & & - & & & \\
\hline Pow Roos E 2011 & 0.024 & $(0.000,0.070)$ & $1 / 42$ & & & & & & \\
\hline Phoa KN 2016 & 0.007 & $(0.000,0.028)$ & $0 / 67$ & & & & & & \\
\hline Pouw RE 2018 & 0.009 & $(0.000,0.019)$ & $3 / 332$ & & - & & & & \\
\hline \multirow[t]{3}{*}{ Overall $\left(I^{\wedge} 2=0 \%, P=0.869\right)$} & 0.009 & $(0.001,0.018)$ & $4 / 453$ & & & & & & \\
\hline & & & & 0 & 0.02 & 0.04 & 0.06 & 0.08 & $\widehat{0.1}$ \\
\hline & & & & \multicolumn{6}{|c|}{ Proportion } \\
\hline
\end{tabular}

Supplementary Figure 6 Sensitivity analysis focused on prospective studies: perforation rate (Forest plot) CI, Confidence Interval; Ev, events; Trt, treatments 


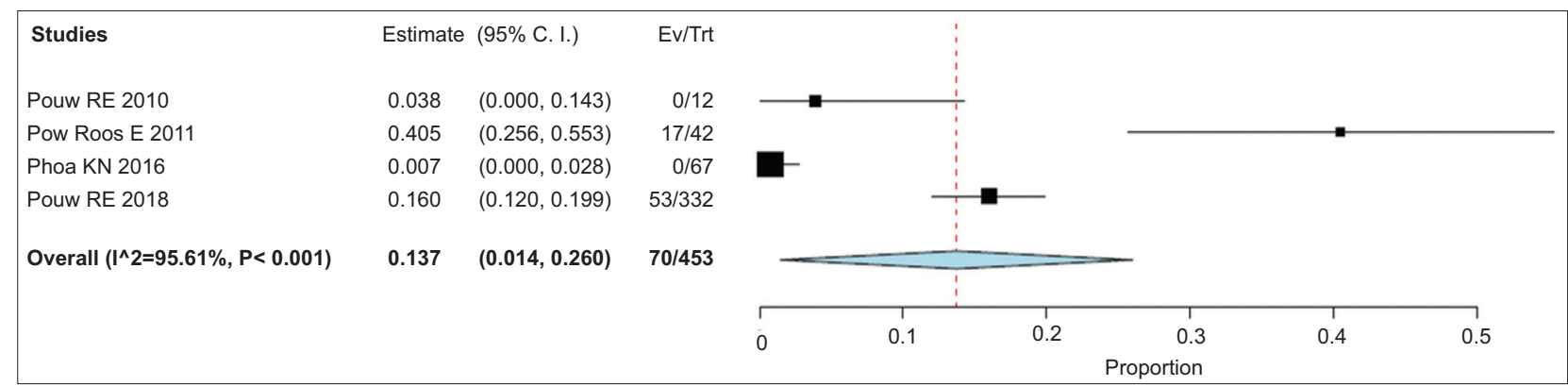

Supplementary Figure 7 Sensitivity analysis focused on prospective studies: overall bleeding rate (Forest plot)

CI, Confidence Interval; Ev, events; Trt, treatments

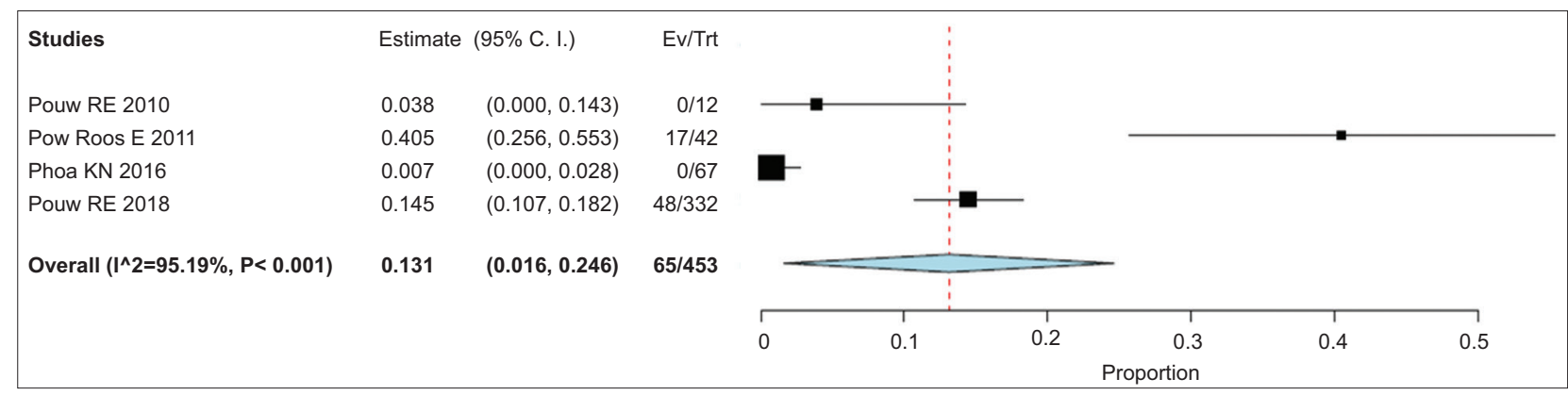

Supplementary Figure 8 Sensitivity analysis focused on prospective studies: intraprocedural bleeding rate (Forest plot) CI, Confidence Interval; Ev, events; Trt, treatments

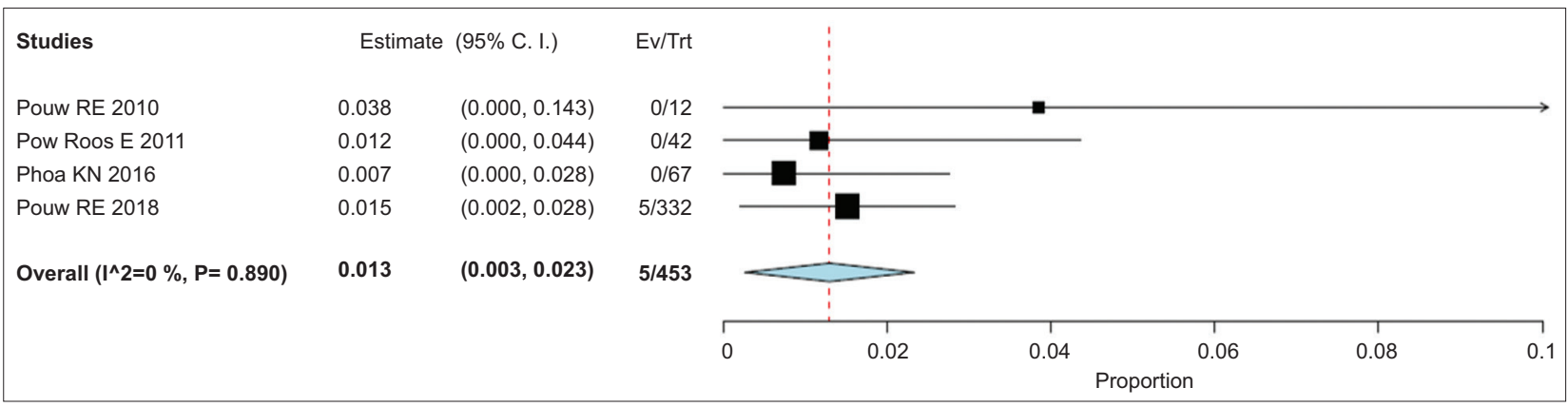

Supplementary Figure 9 Sensitivity analysis focused on prospective studies: postprocedural bleeding rate (Forest plot) CI, Confidence Interval; Ev, events; Trt, treatments

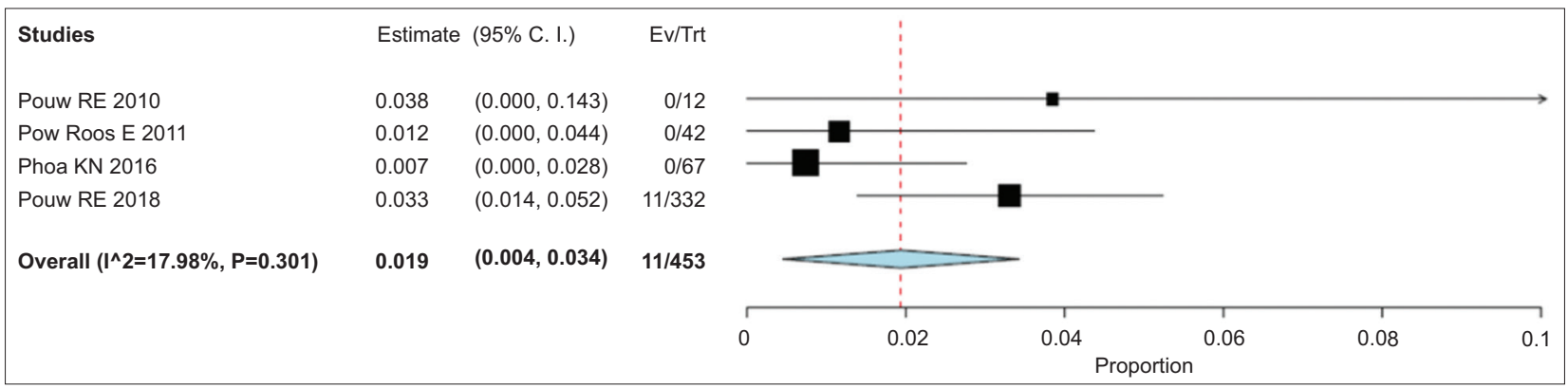

Supplementary Figure 10 Sensitivity analysis focused on prospective studies: stricture rate (Forest plot) CI, Confidence Interval; Ev, events; Trt, treatments 


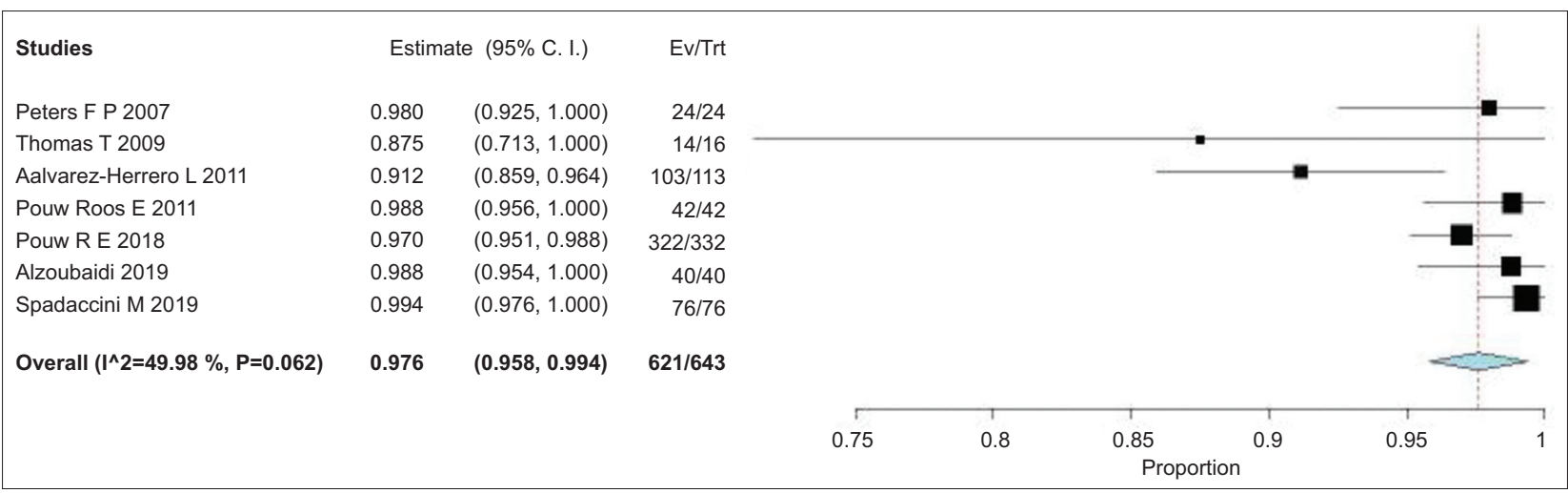

Supplementary Figure 11 Forest plot: complete resection rate

CI, Confidence Interval; Ev, events; Trt, treatments

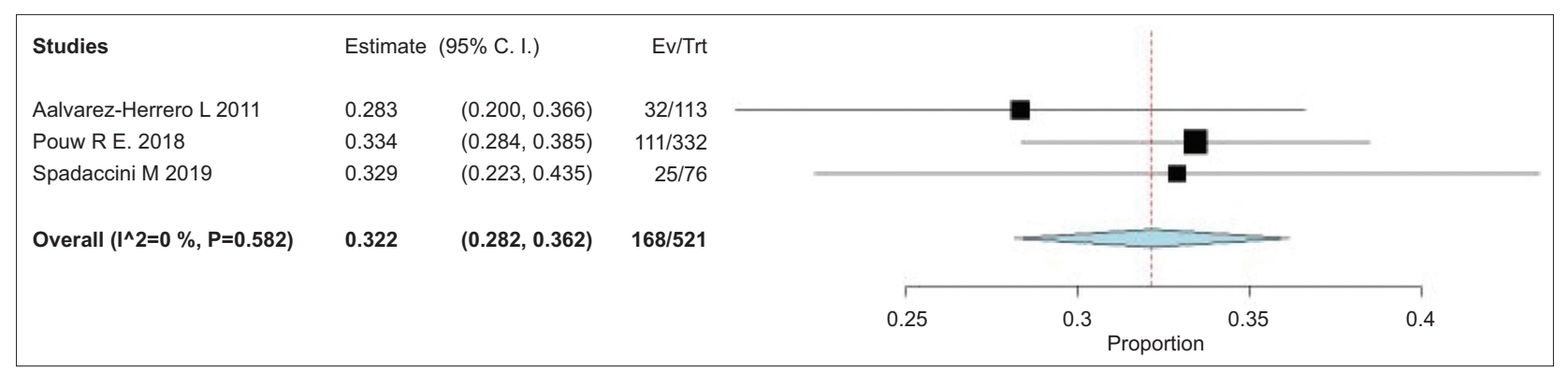

Supplementary Figure 12 Forest plot: en bloc resection rate

CI, Confidence Interval; Ev, events; Trt, treatments

\begin{tabular}{|c|c|c|c|c|c|c|c|c|c|}
\hline Studies & Estimate & (95\% C. I.) & $\mathrm{Ev} / \mathrm{Trt}$ & & & & & & \\
\hline Peters F P 2007 & 0.980 & $(0.925,1.000)$ & $24 / 24$ & & & & & & \\
\hline Thomas T 2009 & 0.750 & $(0.538,0.962)$ & $12 / 16$ & & & & & & \\
\hline Brahmania M 2010 & 0.568 & $(0.408,0.727)$ & $21 / 37$ & & & 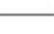 & & & \\
\hline Pouw Roos E 2011 & 0.988 & $(0.956,1.000)$ & $42 / 42$ & & & & & & \\
\hline Pouw R E 2018 & 0.979 & $(0.963,0.994)$ & $325 / 332$ & & & & & & \\
\hline Alzoubaidi D 2019 & 0.900 & $(0.807,0.993)$ & $36 / 40$ & & & & & 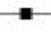 & \\
\hline Spadaccini M 2019 & 0.974 & $(0.938,1.000)$ & $74 / 76$ & & & & & & \\
\hline \multirow[t]{3}{*}{ Overall $\left(\left.\right|^{\wedge} 2=81.71 \%, P<0.001\right)$} & 0.941 & $(0.899,0.984)$ & $534 / 567$ & & & & & & \\
\hline & & & & 0.5 & 0.6 & 0.7 & 0.8 & 0.9 & 1 \\
\hline & & & & \multicolumn{6}{|c|}{ Proportion } \\
\hline
\end{tabular}

Supplementary Figure 13 Forest plot: R0 resection rate

CI, Confidence Interval; Ev, events; Trt, treatments 